\title{
Reconstruction and Simulation of Stratospheric Ozone Distributions during the 2002 Austral Winter
}

\author{
C. E. Randall, ${ }^{*}$ G. L. Manney, ${ }^{+}, \#$ D. R. Allen, ${ }^{\#}$ R. M. Bevilacqua, ${ }^{\#}$ J. Hornstein, ${ }^{\#}$ C. Trepte, ${ }^{@}$ \\ W. LAhoz, \& J. AJTIC, ** AND G. BodEKER ${ }^{+}+$ \\ *Laboratory for Atmospheric and Space Physics, University of Colorado, Boulder, Colorado \\ ${ }^{+}$Jet Propulsion Laboratory, California Institute of Technology, Pasadena, California \\ \#Naval Research Laboratory, Washington, D.C. \\ ${ }^{\circledR}$ Langley Research Center, Hampton, Virginia \\ \&Data Assimilation Research Centre, Reading, United Kingdom \\ **National Institute of Water and Atmospheric Research, Central Otago, and Department of Physics and Astronomy, \\ University of Canterbury, Christchurch, New Zealand \\ ${ }^{+}+$Department of Physics and Astronomy, University of Canterbury, Christchurch, New Zealand
}

(Manuscript received 12 June 2003, in final form 17 December 2003)

\begin{abstract}
Satellite-based solar occultation measurements during the 2002 austral winter have been used to reconstruct global, three-dimensional ozone distributions. The reconstruction method uses correlations between potential vorticity and ozone to derive "proxy" distributions from the geographically limited occultation observations. Ozone profiles from the Halogen Occultation Experiment (HALOE), the Polar Ozone and Aerosol Measurement III (POAM III), and the Stratospheric Aerosol and Gas Experiment II and III (SAGE II and III) are incorporated into the analysis. Because this is one of the first uses of SAGE III data in a scientific analysis, preliminary validation results are shown. The reconstruction method is described, with particular emphasis on uncertainties caused by noisy and/or multivalued correlations. The evolution of the solar occultation data and proxy ozone fields throughout the winter is described, and differences with respect to previous winters are characterized. The results support the idea that dynamical forcing early in the 2002 winter influenced the morphology of the stratosphere in a significant and unusual manner, possibly setting the stage for the unprecedented major stratospheric warming in late September. The proxy is compared with ozone from mechanistic, primitive equation model simulations of passive ozone tracer fields during the time of the warming. In regions where chemistry is negligible compared to transport, the model and proxy ozone fields agree well. The agreement between, and changes in, the large-scale ozone fields in the model and proxy indicate that transport processes, particularly enhanced poleward transport and mixing, are the primary cause of ozone changes through most of the stratosphere during this unprecedented event. The analysis culminates with the calculation of globally distributed column ozone during the major warming, showing quantitatively how transport of low-latitude air to the polar region in the middle stratosphere led to the diminished ozone hole in 2002.
\end{abstract}

\section{Introduction}

The 2002 Southern Hemisphere (SH) winter was marked by unusually high levels of dynamical activity that led to a stratospheric major warming in mid- to late September (e.g., Allen et al. 2003; Hoppel et al. 2003; Sinnhuber et al. 2003; Weber et al. 2003). Analyses of meteorological parameters throughout the winter show

\footnotetext{
\#\# Additional affiliation: Department of Natural Sciences, New Mexico Highlands University, Las Vegas, New Mexico.
}

Corresponding author address: Dr. Cora E. Randall, Laboratory for Atmospheric and Space Physics, University of Colorado, UCB 392, Boulder, CO 80309-0392.

E-mail: cora.randall@lasp.colorado.edu that increased wave activity forced from the troposphere occurred as early as May (e.g., Newman and Nash 2005; Scaife et al. 2005). During the major warming, the middle-stratospheric polar vortex elongated, moved off of the pole, and split in two before recovering to a smaller and weaker pole-centered vortex in early October. The warming was accompanied by rapid, large-scale transport of air from the subtropics to the polar region (e.g., Charlton et al. 2005; Manney et al. 2005). This brought ozone-rich air to the polar region, as seen by the Polar Ozone and Aerosol Measurement III (POAM III) instrument (Hoppel et al. 2003; Allen et al. 2003). Photochemical ozone loss in the lower stratosphere ceased in late September presumably because of the higher temperatures and consequent lack of polar stratospheric cloud (PSC) formation (Hoppel 
et al. 2003; Nedoluha et al. 2003), but the lower stratospheric vortex remained intact, effectively containing ozone-depleted air.

In this paper, we examine how the unique dynamical situation in 2002 was reflected in ozone profile measurements from solar occultation instruments and use these data to explore the effects of the major warming on hemispheric ozone distributions. We focus on the time period from 1 May through 31 October 2002 and also compare these measurements to those in 1999. Solar occultation data are well known for their high accuracy, vertical resolution, and stability. On the other hand, they have sparse geographic coverage, sampling only a very narrow range of latitudes in each hemisphere on any given day. It is therefore useful to combine data from multiple instruments to obtain broader coverage. In this paper, we apply potential vorticity (PV) mapping (e.g., Randall et al. 2002) to reconstruct semiglobal ozone fields with data from the four dedicated solar occultation instruments in orbit in 2002. These include the Stratospheric Aerosol and Gas Experiment (SAGE) II (data version 6.1) and III (version 2.00), the Halogen Occultation Experiment (HALOE, version 19) and POAM III (v3.0). Section 2 describes the individual solar occultation datasets, and the reconstruction method by which the information contained in these datasets is extended to a global scale. Section 3 expands on the work of Hoppel et al. (2003) and Allen et al. (2003) by showing how the unusual dynamical activity in 2002 was reflected in the solar occultation data themselves, followed by descriptions of the more global effects using hemispheric reconstructions of the combined datasets. Section 4 compares the reconstructed fields with results from a global, mechanistic model (Manney et al. 2005). Based on the reconstructed solar occultation fields and model simulations, section 5 discusses how the altitude distribution of globally distributed ozone resulted in the diminished ozone hole of 2002.

\section{Solar occultation data and reconstruction method}

\section{a. Datasets}

Latitudes of solar occultation measurements vary smoothly in time, with the precise locations depending on the satellite inclination and equator crossing time. The SAGE II/III, HALOE, and POAM datasets in general have complementary latitude sampling, as depicted in Fig. 1 for 2002 and 1999 (1999 was chosen to illustrate a "typical" year because of missing data from SAGE II or HALOE in 2000 and 2001). SAGE II and HALOE both cover a wide range of latitudes in about a 1-month time span, with coverage that is similar but offset in time by about 2 weeks. The latitude coverage ranges from about $55^{\circ}-60^{\circ} \mathrm{S}$ to the equator from May to October, with the exception of one $\sim$ week-long excur-
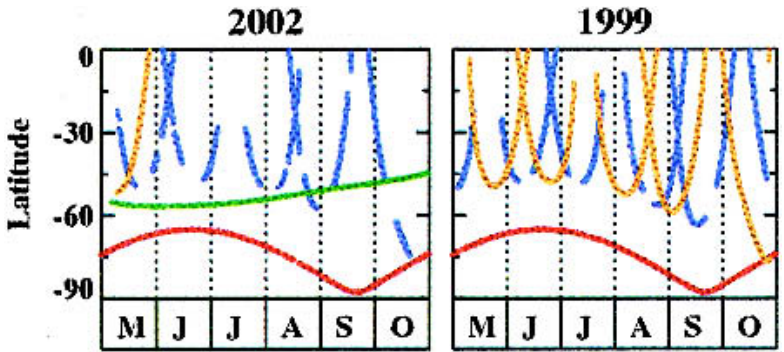

FIG. 1. Latitudes of measurements from May through Oct in (left) 2002 and (right) 1999 from the SAGE II (orange), SAGE III (green), HALOE (blue), and POAM (red) solar occultation instruments.

sion to polar latitudes by HALOE (SAGE II) in October of 2002 (1999). The SAGE II instrument is still operational, but data are temporarily unavailable after May 2002 because of data-processing problems. POAM III measurement latitudes repeat annually and in the $\mathrm{SH}$ range between about $63^{\circ}$ and $88^{\circ} \mathrm{S}$, reaching the most poleward latitude near equinox. The SAGE III instrument was launched in December 2001, and data production began in May 2002. From May through October 2002, SAGE III measurements occurred between about $55^{\circ}$ and $45^{\circ} \mathrm{S}$.

The SAGE II, HALOE, and POAM III ozone measurements have been validated previously (e.g, Brühl et al. 1996; Cunnold et al. 1989; Morris et al. 2002; Randall et al. 2003, and references therein) and are known to be of excellent quality in the stratospheric regions discussed here. SAGE III data are currently undergoing initial validation, so here we present some comparisons with the other solar occultation datasets to establish the validity of SAGE III data for the current work. Figure 2 compares SAGE III data in 2002 with coincident observations from POAM and HALOE. Coincidences included measurements that occurred within $500 \mathrm{~km}$ at the same local sunrise or sunset. These occurred only at local sunset, and only in the Northern Hemisphere $(\mathrm{NH})$ for the POAM data and in the $\mathrm{SH}$ for the HALOE data. All 89 POAM coincidences occurred between 31 August and 21 September, with an average separation of $406 \pm 69 \mathrm{~km}$. All 33 HALOE coincidences occurred between 23 August and 9 September, with an average separation of $344 \pm 125 \mathrm{~km}$. The comparisons in Fig. 2 are restricted to the altitude range between 15 and $40 \mathrm{~km}$, the region of interest for the present work. The agreement is within $\pm 10 \%$ at most of these altitudes, and is often within $\pm 5 \%$, clearly indicating that the SAGE data are appropriate for use in this work. Figure 2 also indirectly shows the very good agreement between POAM and HALOE, discussed in detail by Randall et al. (2003). Although differences between the measurements are relatively small, they are taken into account in the analyses below. 


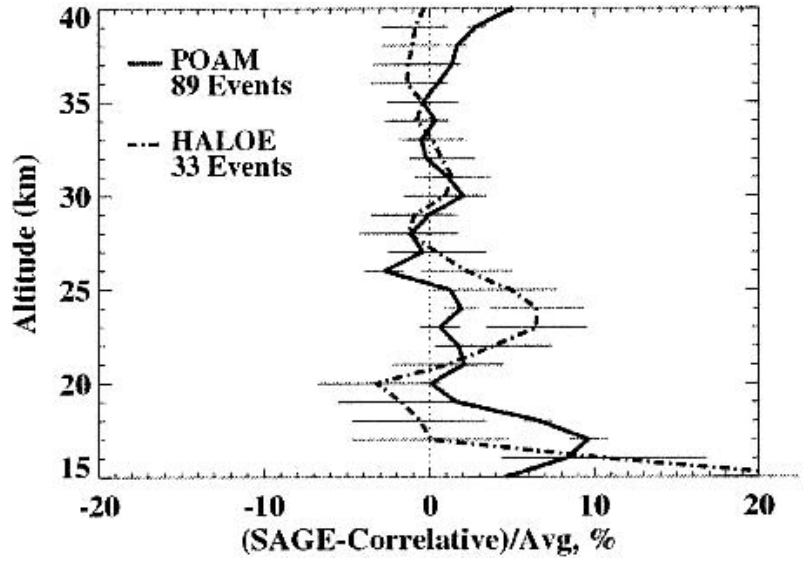

FIG. 2. Average differences between SAGE III and POAM (solid line) or HALOE (dot-dash line) for coincident measurements in 2002. "Error" bars denote the statistical uncertainty in the mean differences, defined by the standard deviation of the differences at each altitude divided by the square root of the number of comparisons (see, e.g., Randall et al. 2003).

\section{b. $3 D$ reconstruction method}

To move beyond the analysis of individual solar occultation datasets, our goal is to combine the datasets in a manner that preserves information regarding the geophysical state of the atmosphere. Formal data assimilation based on our knowledge of global transport and chemistry is a sophisticated and complex method for accomplishing this, and such techniques are capable of incorporating profile data from limb sounders (e.g., Struthers et al. 2002; Fonteyn et al. 2003, manuscript submitted to J. Atmos. Sci.). Alternatively, the PVmapping technique, established more than a decade ago (e.g., Butchart and Remsberg 1986; Schoeberl et al. 1989), provides a simpler yet effective way to reconstruct global fields from geographically sparse measurements (e.g., Randall et al. 2002, and references therein). This approach, which provides a reasonable, efficient, and direct method of combining these high vertical resolution measurements, is the one used here. The premise behind the mapping technique is that at any given point in time, a long-lived trace species, $\chi$, forms a single compact isentropic relationship with dynamical tracers such as PV or the related quantity, equivalent latitude (EL), the latitude at which a PV contour would reside if conservatively conformed to a pole-centered circle (e.g., Leovy et al. 1985; Allaart et al. 1993; Lary et al. 1995; Manney et al. 1999). If this relationship is determined by measuring $\chi$ over a sufficient range of $\mathrm{PV} /$ potential temperature $(\theta)$ space, it can be used to derive $\chi$ at geographic locations outside the actual measurement field, as long as the PV and $\theta$ values at those locations are known. The field so derived is referred to as the "proxy" field in this paper.

For the work described here, two different dynamical parameters were employed, PV and the recently devel- oped tracer equivalent latitude (TREL Allen and Nakamura 2003). Isentropic PV fields (and resulting EL) were calculated from daily 1200 UTC Met Office meteorological analyses on a $2.5^{\circ} \times 3.75^{\circ}$ (latitudelongitude) grid (Swinbank and O'Neill 1994). Here, EL is referred to as "low" or "high" based only on its magnitude, not sign. Thus, low EL refers to tropical air, and high EL refers to polar air, even though EL is negative in the SH. TREL is based on a numerical synthesis of a PV-like tracer by solving the advection-diffusion problem on isentropic surfaces (Haynes and Shuckburgh 2000; Allen and Nakamura 2001). It is qualitatively similar to EL calculated from PV, for instance ranging from $-90^{\circ}$ to $90^{\circ}$. TREL has been used successfully to investigate record low column ozone over Europe on 30 November 1999, ozone loss during the 1999-2000 Arctic winter, and the Arctic vortex breakup in late winter/ spring of 1994 (Allen and Nakamura 2002, 2003). Because the TREL- and PV-mapping techniques are fundamentally based on the same concepts, they give qualitatively similar results. Nevertheless, there are differences in detail that are useful for evaluating drawbacks to either of the methods. A comprehensive cross comparison of the TREL and PV reconstructions is beyond the scope of this paper. Since our previous work (Randall et al. 2002) was based on PV reconstructions, we primarily show results from the PV proxy here; however, we also describe instances where differences between the PV and TREL proxy results indicate potential errors in the reconstructed fields.

Reconstruction of proxy ozone follows Randall et al. (2002), with several notable improvements. First, the previous work incorporated only POAM data in the analysis, while the current work incorporates data from HALOE, SAGE II and III, and POAM. Inclusion of multiple datasets requires strict attention to differences between measurements from the different instruments. To address this, normalization profiles were calculated and applied to the different datasets. These profiles were derived from the validation intercomparison results presented by Randall et al. (2003), as well as from comparisons such as those shown in Fig. 1 (for SAGE III). POAM profiles were normalized to sonde data below $850 \mathrm{~K}$, and all of the other satellite data were normalized to the corrected POAM profiles. Normalization corrections for all instruments were on the order of $5 \%$ or less at all theta levels except for SAGE III, where normalization factors between 450 and $600 \mathrm{~K}$ were $\sim 7 \%$. Second, Randall et al. (2002) used quadratic functions to determine the $\mathrm{PV}-\mathrm{O}_{3}$ relations. While this works well for a single dataset, quadratic functions are inadequate to characterize the $\mathrm{PV}-\mathrm{O}_{3}$ relation when geographically dispersed measurements are included. The analysis here utilizes a nine-node cubic spline fit, where the number of nodes was determined via trial and error. Third, the occultation data were smoothed in PV or TREL space before calculating the spline fit to remove spurious, random data 
points that distorted the fit. Fourth, the inclusion of midlatitude and tropical data, and our focus on the $\mathrm{SH}$ equinox timeframe in late September, necessitated that a method be developed to handle latitudinally varying $\mathrm{PV}-\mathrm{O}_{3}$ relations. This is discussed in more detail below.

The mapping technique is illustrated in Fig. 3 for 30 September 2002 , for the $1050-\mathrm{K}$ potential temperature surface. As noted above, successful PV mapping requires measurements over a wide range of $\mathrm{PV} / \theta$ space. To accomplish this, we use data in a multiple-day window centered on the analysis date. Sensitivity tests (not shown) determined that a 7-day window generally provides reasonable data coverage while avoiding smearing caused by temporal variation in the $\mathrm{PV}-\mathrm{O}_{3}$ relations. Figure 3 shows $\mathrm{O}_{3}$ measurements versus PV (interpolated in time and space to the measurement locations) for 27 September-3 October. During this time period, SH HALOE data were acquired at geographic latitudes equatorward of $35^{\circ} \mathrm{S}$, SAGE data were acquired near $49^{\circ} \mathrm{S}$, and POAM data were acquired near $86^{\circ} \mathrm{S}$. Thus, the three satellite instruments sampled very different, but fairly narrow, ranges of solar zenith angles. The EL sampling was much broader, however, as indicated by the span of PV values associated with the data.

Attempts were first made to fit all of the data with a single cubic spline, shown by the yellow symbols. This is problematic, however, since the same value of PV is often associated with multiple values of ozone, so the calculated fit poorly represents the observations. A

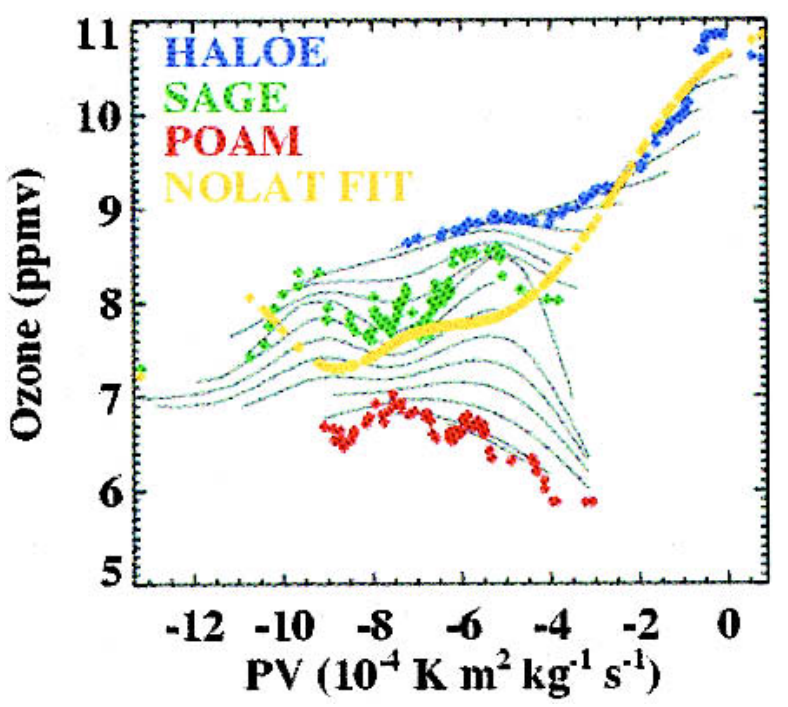

FIG. 3. Measurements of ozone on the $1050-\mathrm{K}$ potential temperature surface from SAGE III (green), HALOE (blue), and POAM (red) vs PV for the 7-day time period centered on $30 \mathrm{Sep}$. Data were smoothed in PV with an 11-point boxcar filter. The yellow points depict a nine-node cubic spline fit to all of the data. Gray lines show the latitude-dependent $\mathrm{PV}-\mathrm{O}_{3}$ relations used to reconstruct proxy ozone fields from the global PV values, for latitudes ranging from $85^{\circ} \mathrm{S}$ (bottom curve) to the equator in $5^{\circ}$ increments. number of different factors may contribute to these multiple-valued $\mathrm{PV}-\mathrm{O}_{3}$ relations, including errors in the $\mathrm{PV}$ fields and time-varying $\mathrm{PV}-\mathrm{O}_{3}$ relations. The primary cause, however, is latitude-dependent photochemistry that changes the $\mathrm{PV}-\mathrm{O}_{3}$ correlations for data acquired at different latitudes. To confirm this explanation, the "latitude history" of the air sampled by the occultation instruments was explored using the 3D trajectory code described by Manney et al. (1994). Tenday back trajectories were run from the occultation locations at the proxy $\theta$ levels where multiple valued PV$\mathrm{O}_{3}$ relations were observed to determine the latitudes and potential temperatures that the parcels had experienced in the previous days. In general, these histories are qualitatively consistent with the interpretation proposed above, in that measurements at high EL with higher ozone have more recently spent most of their time at low latitudes, and those with lower ozone at high latitudes. For example, it was found that on days and at theta levels where SAGE and POAM data agreed, air parcels at the two instrument locations had similar latitude histories; dissimilar latitude histories corresponded to those times and altitudes where measurements from the two instruments disagreed, even for air parcels at the same PV.

To account for the latitude dependence in the $\mathrm{PV}-\mathrm{O}_{3}$ relations, separate nine-node cubic splines are calculated when datasets from different instruments are separated by more than $10^{\circ}$ in latitude. Linear interpolation of the fits in latitude bins corresponding to the resolution of the dynamical tracer field $\left(2.5^{\circ}\right.$ for $\mathrm{PV}$; $1.7^{\circ}$ for TREL) was employed to fill in the latitude regions between the different datasets. In this manner, a systematic $\mathrm{PV}-\mathrm{O}_{3}$ relationship is preserved at all latitudes, but it varies to account for nonconservative processes such as photochemistry. The method neglects the fact that photochemical effects in the $\mathrm{PV}-\mathrm{O}_{3}$ relation should actually vary not according to the instantaneous latitude, but to the latitude (solar insolation) history of the air parcel. This is tantamount to assuming that the air is in chemical equilibrium, in which case the instantaneous latitude would be sufficient to describe the history. It also assumes that any photochemical effects vary linearly with latitude, whereas in theory this is not strictly true. Nevertheless, we use the latitudedependent fits as a reasonable first-order correction to the global fit. In latitude regions poleward of the most poleward occultation measurement or equatorward of the most equatorial occultation measurement, fits were set equal to those at the boundary latitudes.

The gray lines in Fig. 3 show the smoothly varying $\mathrm{PV}-\mathrm{O}_{3}$ relations for the latitudes bounded by the occultation measurements and for latitudes in between. This figure shows that the latitude-dependent fits better represent the occultation data than the single, latitudeindependent fit. Although not shown, these fits converge as expected at low altitudes where photochemical 
time scales are relatively long. This is also true in the ozone hole region (e.g., late September below $550 \mathrm{~K}$ ), where photochemical ozone depletion is confined to vortex air and thus to a large extent correlates with PV. The fitting procedure is most limited where dynamical and chemical time scales are comparable. For example, in dynamically active situations such as a major warming, air drawn up to high latitudes typically changes latitude continuously and rapidly, and thus never reaches chemical equilibrium. In these cases, while the proxy will match well the original occultation data, it can still misrepresent the ozone field in regions that are not directly observed.

\section{c. Proxy validation}

The proxy fields are evaluated in a number of different ways. These include comparisons with the original occultation data used to calculate the proxy, comparisons of the PV and TREL proxy calculations with one another, comparisons with independent data (e.g., ozonesondes), and comparisons with model calculations. Comparisons with the original occultation data might seem redundant, but differences can arise from the various error sources discussed in the previous section. Certainly, if the proxy cannot be trusted at the measurement locations themselves, then its use for deriving conclusions pertaining to other locations is limited. In this section, we discuss statistical comparisons of the proxy fields with the occultation data itself, and with sonde data. Further confirmation of the validity of the proxy is given in later sections within the context of discussions regarding the dynamical influences on global ozone in 2002. Detailed comparison of the two different proxy products is left for future work.
Figures 4 and 5 quantify the differences between the occultation data and the PV proxy fields interpolated to the occultation measurement locations. In the first row of Fig. 4, solar occultation mixing ratios at $\theta=850 \mathrm{~K}$ are plotted along with the interpolated proxy, while the second row shows the differences. With few exceptions, the proxy mixing ratios represent the occultation data very well. Differences are mostly less than $\pm 20 \%$, with agreement often within $\pm 10 \%$. The points in the difference time series are color coded according to EL and confirm that there are no significant systematic differences that depend on EL. The proxy results do show noticeably less variation than the occultation data, as is particularly evident in the POAM and SAGE ozone time series (top row) during September and October. This is a direct result of the cubic-spline-fitting procedure, which removes a substantial amount of scatter from the data. The occultation data have a precision on the order of a few percent, so larger reductions in scatter indicate a loss of information in the proxy.

Figure 5 shows the average differences from 450 to $1500 \mathrm{~K}$ for all of the data from 1 May through $31 \mathrm{Oc}$ tober 2002 . From 500 to $1500 \mathrm{~K}$, the average agreement between the proxy and the data is excellent. The one- $\sigma$ spread in the differences is only at the $10 \%$ level, leading to mean uncertainties in the average differences of less than $1 \%$. At $450 \mathrm{~K}$, the differences for the SAGE and HALOE measurement locations are larger, but they are still less than $10 \%$. Although not shown, the TREL proxy results are also biased high with respect to the SAGE and HALOE data at $450 \mathrm{~K}$. The reason for this is currently undetermined and is the subject of future work.

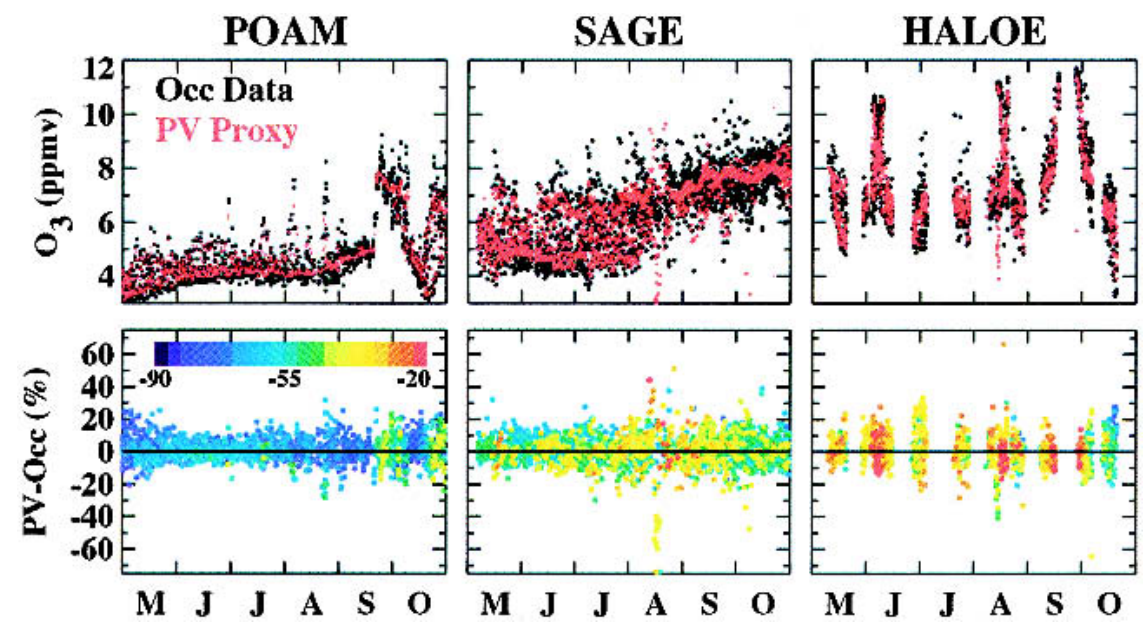

FIG. 4. Comparison of PV proxy calculations and occultation data at $850 \mathrm{~K}$ from May through Oct 2002. (top) Time series of occultation data (black) and the PV proxy ozone fields (red) interpolated to the (left) POAM, (center) SAGE III, and (right) HALOE measurement locations. (bottom) Differences between the PV proxy and occultation data, relative to the occultation data. Differences are color coded according to the associated EL value at the measurement locations using the color scale given in the bottom left. 


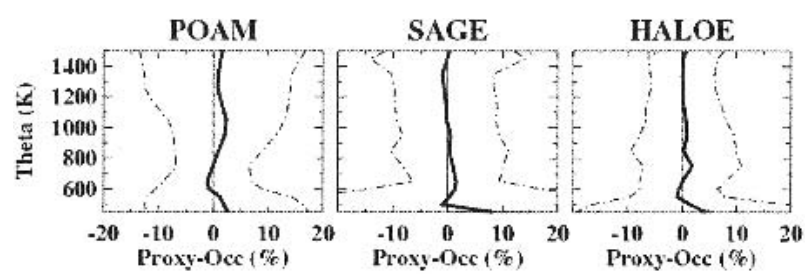

FIG. 5. Average differences (solid lines) between the occultation data (Occ) and the PV proxy interpolated to the (left) POAM, (center) SAGE III, and (right) HALOE occultation locations, for all measurements from 1 May through 31 Oct 2002. One-sigma standard deviations of the distributions are given by the dot-dash lines; $1 \sigma$ uncertainties in the mean differences are approximately equal to the width of the solid lines.

A more stringent test of the proxy is to compare it to independent measurements. Orsolini et al. (2005) show qualitative agreement between the PV proxy fields and Michelson Interferometer for Passive Atmospheric Sounding (MIPAS) data in October and November 2002. Here we compare the PV proxy ozone profiles to sonde data from two different SH stations for the period 1 May-31 October 2002. During this period, there were 34 measurements made at the South Pole [operated by the National Oceanic and Atmospheric Administration (NOAA) Climate Monitoring and Diagnostics Laboratory] and 26 measurements at Lauder, New Zealand $\left(45^{\circ} \mathrm{S}, 169.7^{\circ} \mathrm{E}\right.$, operated by the National Institute of Water and Atmospheric Research). Proxy fields corresponding to the dates of the sonde measurements were interpolated to these sonde station locations. Qualitatively, as shown in Fig. 6 (top), the proxy and sonde data are in excellent agreement. In particular, note that the wide range of values observed at the South Pole is captured well by the proxy. For instance, ozone mixing ratios below about $600 \mathrm{~K}$ hover around 2.5-3 ppmv early in the winter but drop to less than 1 ppmv in late September when photochemical ozone depletion occurs; this is observed in both the sonde and proxy profiles. Above $600 \mathrm{~K}$, there are several sonde profiles with mixing ratios that maximize near 6-7 ppmv in late September and early October, indicative of the transport of low-latitude, ozone-rich air to the pole; this too is captured by the proxy fields.

More quantitatively, the average differences between the proxy and sonde data are within $\pm 10 \%$ except below $500 \mathrm{~K}$ at South Pole, where they reach $+15 \%$; this positive bias is thus consistent with the bias found in comparisons to the solar occultation data themselves. Within the statistical uncertainties of the comparisons, however, the differences at most altitudes are negligible, or nearly so. This agreement is quite satisfactory and nearly matches the statistical agreement observed between the sondes and the individual occultation datasets themselves. The standard deviations of the distribution of differences can be relatively large, particularly at The South Pole, with most values between 30 and $50 \%$. This is expected since, as discussed above, the fitting procedure smoothes through any variations at a given EL.

\section{Dynamical influences on ozone in 2002}

\section{a. Solar occultation observations}

In this section, we discuss how the unusual dynamics in 2002 influenced the ozone distributions observed by the solar occultation instruments. Figure 7 presents POAM, SAGE III, and HALOE ozone mixing ratios from May through October of 2002 on three $\theta$ surfaces. In most years, POAM measurements throughout the winter and early spring are made at high EL values, poleward of $\mathrm{EL} \sim 60^{\circ} \mathrm{S}$. In late September of 2002, however, POAM sampled air with lower EL, and significantly higher ozone mixing ratios, at all three $\theta$ levels. This phenomenon, which is caused by enhanced descent and poleward transport of subtropical air during the major warming, has been described in detail by Hoppel et al. (2003) and Allen et al. (2003). At $500 \mathrm{~K}$, POAM ozone increases in May through June because of enhanced descent, then decreases during August and September via photochemical ozone depletion; overall, this behavior is similar to that seen in other years. However, whereas in other years 500-K POAM ozone mixing ratios reached values near 0 ppmv in late September, the decline in 2002 was mostly arrested by the

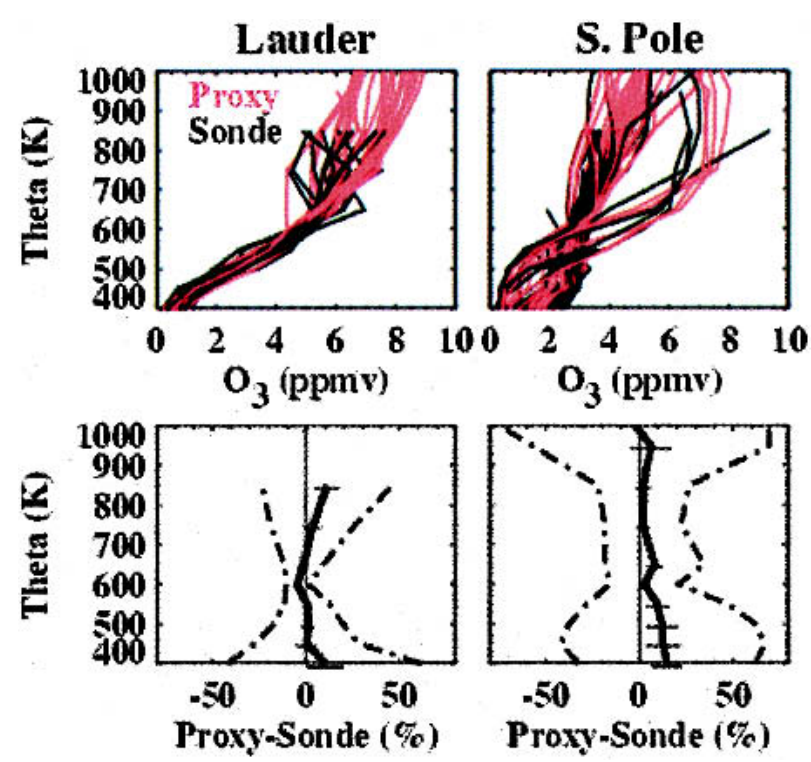

FIG. 6. Comparison of ozonesonde profiles (black) measured at (left) Lauder and (right) the South Pole to the PV proxy profiles (red) interpolated to the sonde locations: (top) all sonde profiles acquired at the stations between 1 May and 31 Oct 2002 (26 at Lauder; 34 at South Pole), along with the corresponding interpolated proxy profiles and (bottom) the average \% differences (solid lines); error bars denote the $1 \sigma$ uncertainty in the mean differences, and dash-dot lines denote 1 std dev of the distribution. 

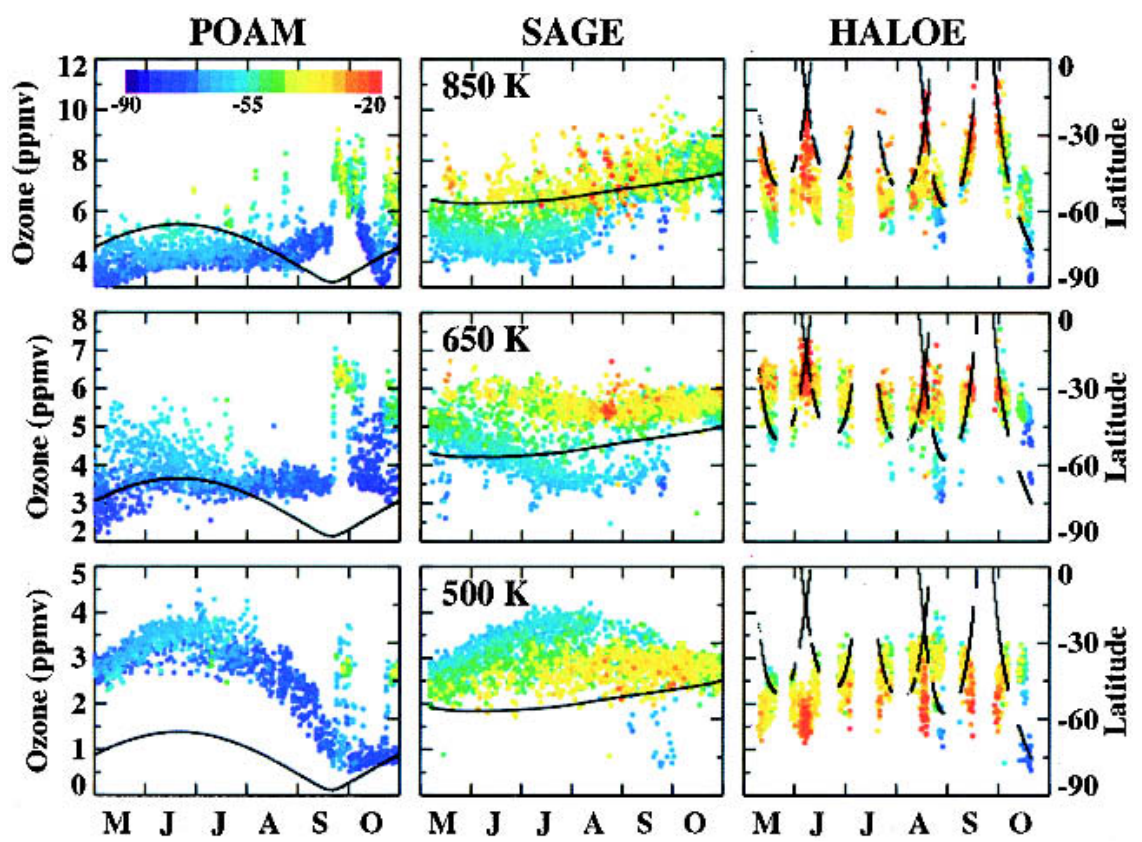

FIG. 7. Time series of ozone mixing ratios in ppmv (left axis) from (left) POAM, (center) SAGE, and (right) HALOE on potential temperature surfaces at (top) 850, (middle) 650, and (bottom) $500 \mathrm{~K}$. Individual dots correspond to individual occultation measurements and are color coded by the EL at the measurement location (top left). Black lines in each panel show the geographic latitudes of the occultation measurements, referenced to the right axis.

major warming, so that even at the highest EL values sampled by POAM, mixing ratios did not decrease below about $0.5 \mathrm{ppmv}$. At $850 \mathrm{~K}$, there is substantial variability in June-August, both in ozone mixing ratios and in EL values, that has never been observed in POAM data from previous years. As described by Allen et al. (2003) and discussed more below, these pulses of high ozone mixing ratios are caused by enhanced wave activity in the middle and upper stratosphere.

The second column in Fig. 7 shows SAGE III observations. Perhaps the most remarkable feature in the SAGE III data is the measurement at $500 \mathrm{~K}$ of high-EL, low-ozone air in late September, when the SAGE III latitudes were near $48^{\circ} \mathrm{S}$. The SAGE III profiles corresponding to these points have the same character as the ozone "hole" profiles typically measured at high latitudes (e.g., by the POAM instrument or South Pole sondes) at this time but normally not seen at such a low latitude. In general at $500 \mathrm{~K}$, SAGE samples both in and out of the vortex through September, with higher mixing ratios observed inside the vortex (prior to chemical ozone depletion) as ozone-rich air descends from higher altitudes.

SAGE also samples in and out of the vortex at 650 and $850 \mathrm{~K}$, but at these $\theta$ levels ozone mixing ratios are lower inside the vortex throughout the winter. Since enhanced diabatic descent inside the vortex at these altitudes has less effect on ozone mixing ratios, these differences are due largely to latitude (solar insolation) gradients in ozone and the isolation of vortex air that maintains and enhances these gradients (Manney et al. 1995; Randall et al. 1995). At all three $\theta$ levels, pulses of ozone lying either above or below the average are apparent in May through August corresponding to excursions in EL. For example, note the low-EL, high-ozone measurements at $850 \mathrm{~K}$ in early July and early, mid-, and late August and the low-EL, low-ozone measurements at $500 \mathrm{~K}$ in June and July. Most pronounced at $650 \mathrm{~K}$ in this regard is the observation of anomalously low ozone corresponding to high-EL air in early to midMay, with mixing ratios comparable to those seen in the high-latitude POAM data.

Also of interest in the SAGE III data is the abrupt decrease in variability, most noticeable at $650 \mathrm{~K}$ but also apparent at $850 \mathrm{~K}$, immediately following the warming. This is caused by the fact that beginning in October, SAGE sampled a slightly narrower range of EL, at the same time that the vortex area had shrunk considerably. To illustrate this, Fig. 8 shows SAGE III ozone mixing ratios at $650 \mathrm{~K}$ as a function of EL for 2-week periods from 1 August through 15 October. From August through September, a region of steep ozone gradients is evident, denoting the mixing barrier at the vortex edge region. At the beginning of this time period, the steep ozone gradient is centered around an EL of $55^{\circ} \mathrm{S}$, but by the end of September it is near $65^{\circ} \mathrm{S}$. Since most of the measurements in the first two weeks of October occurred equatorward of $\mathrm{EL}=60^{\circ} \mathrm{S}$, it is not surprising that the time series lacks low-ozone mixing ratios at this time. This is consistent with the analy- 


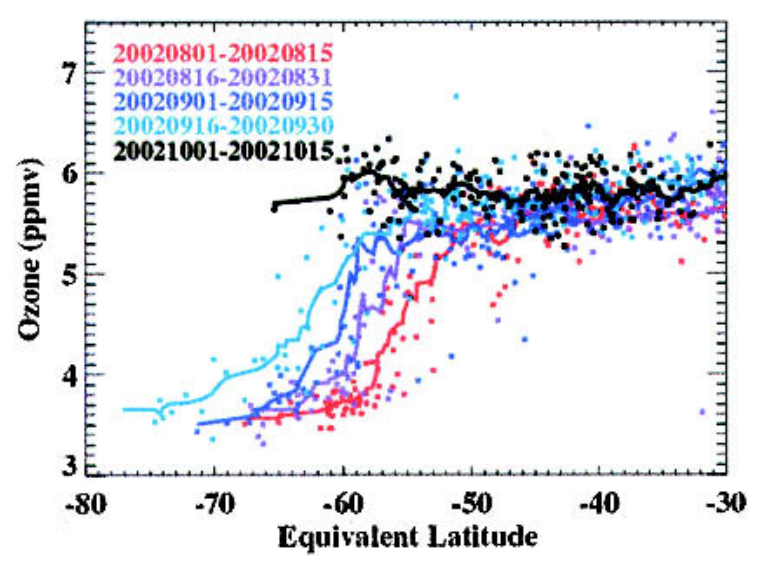

FIG. 8. SAGE III ozone at $650 \mathrm{~K}$ vs EL for the time periods (yyyymmdd) given in the legend. Symbols represent individual SAGE measurements, and lines show the data smoothed with an 11-point boxcar filter.

sis of Allen et al. (2003) who showed that the region (at $850 \mathrm{~K}$ ) of low equivalent length (barrier to mixing) gradually diminished in size throughout the winter. The equatorial edge of the containment region crept poleward in EL space until the warming, at which time the region of mixing temporarily increased dramatically in size, and after which the mixing barrier was situated significantly poleward of its position immediately before the warming.

Superficially, the HALOE data in Fig. 7 show no immediately obvious effects of the increased dynamical activity in 2002 , even when compared to similarly pre- sented data from other years. Because of the large range of latitudes sampled, the HALOE instrument makes measurements over a wide range of EL as well; in general, relative mixing ratios at low and high EL values are consistent with expectations. Nevertheless, because of its low-latitude excursions, the HALOE data provide unique information about ozone distributions that is geographically complementary to the information provided by the other instruments. This information is used below to explore the spatial extent of the more obvious dynamical effects at the higher latitudes.

\section{b. Proxy data at the solar occultation locations}

To further confirm the validity of the proxy fields within the context of the unusual 2002 meteorological conditions, Fig. 9 shows the PV proxy fields interpolated back to the solar occultation measurement locations. The time series in Figs. 7 and 9 are remarkably consistent in terms of their overall characteristics. As expected, the proxy correctly captures the EL gradients in ozone mixing ratios that are particularly prominent in the SAGE and HALOE data. The proxy correctly portrays the sudden increases in ozone observed at the POAM measurement locations as the major warming occurred and the occurrence of anomalously low-ozone mixing ratios at $500 \mathrm{~K}$ at the SAGE locations. The proxy also shows pulses of ozone variability preceding the major warming at the POAM and SAGE measurement locations, especially evident as spikes of high ozone mixing ratios at $850 \mathrm{~K}$, although not of the same magnitude as observed in the occultation data. The

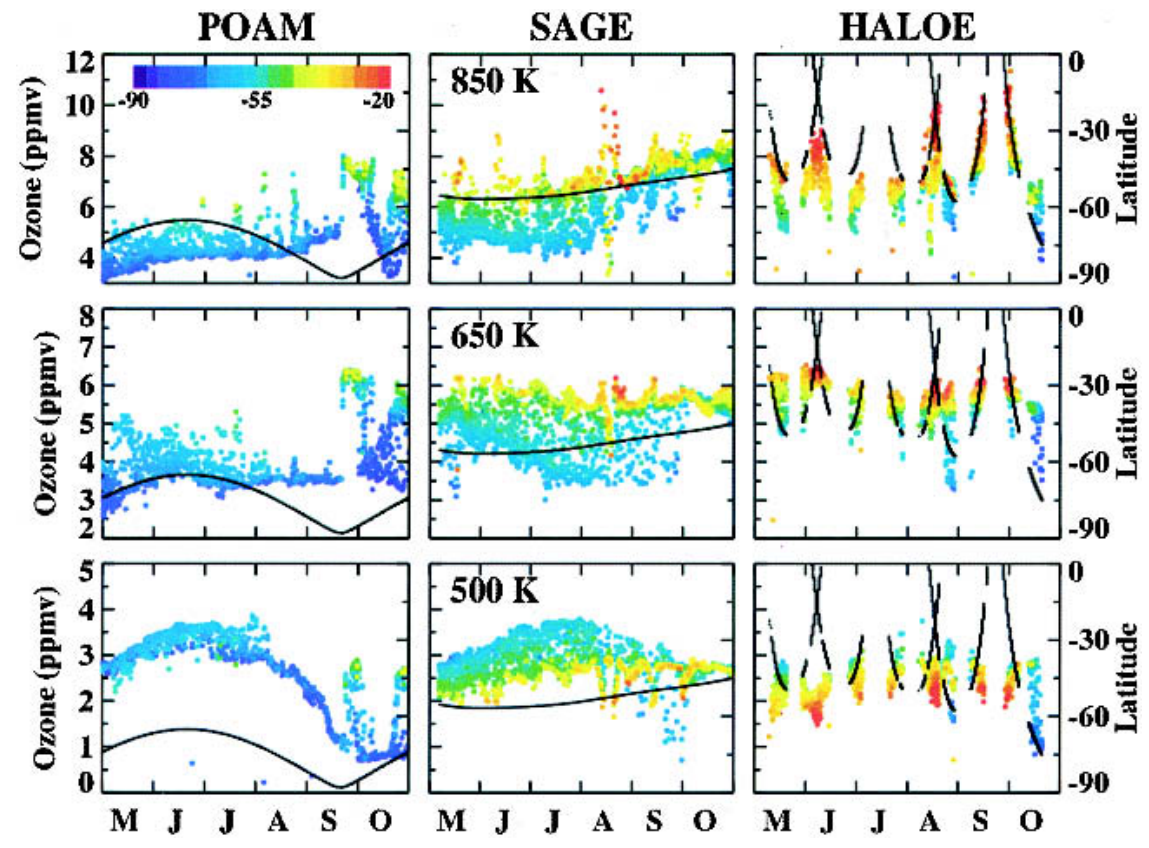

FIG. 9. Same as in Fig. 7, but for the PV proxy data interpolated back to the solar occultation measurement locations. 
anomalous ozone mixing ratios at $850 \mathrm{~K}$ in the SAGE III data in mid-August (both very low and very high ozone mixing ratios at relatively low EL) require further investigation; these deviations, while present, were much reduced in the TREL proxy calculations. The proxy generally exhibits noticeably less variation than the occultation data, as was discussed above.

To more closely examine the reconstruction of episodic increases in variability, Fig. 10 shows the running 7-day standard deviation in ozone mixing ratios from each of the occultation datasets between 1 May and 30 September. Since the measurement locations generally vary by only a few degrees in a 7-day period, to first order these standard deviations can be interpreted as zonal variations in ozone, the magnitude of which is influenced strongly by vortex asymmetry. Episodic enhancements in variability occur in all three datasets, with approximate temporal correlation. Figure 10 also shows the results from the PV proxy interpolated to the occultation locations. Because the proxy variations are smaller than observed by the occultation instruments, they have been multiplied by 1.2 to facilitate the morphological comparisons. Clearly, most of the characteristics of the occultation plots are reproduced well by the proxy.

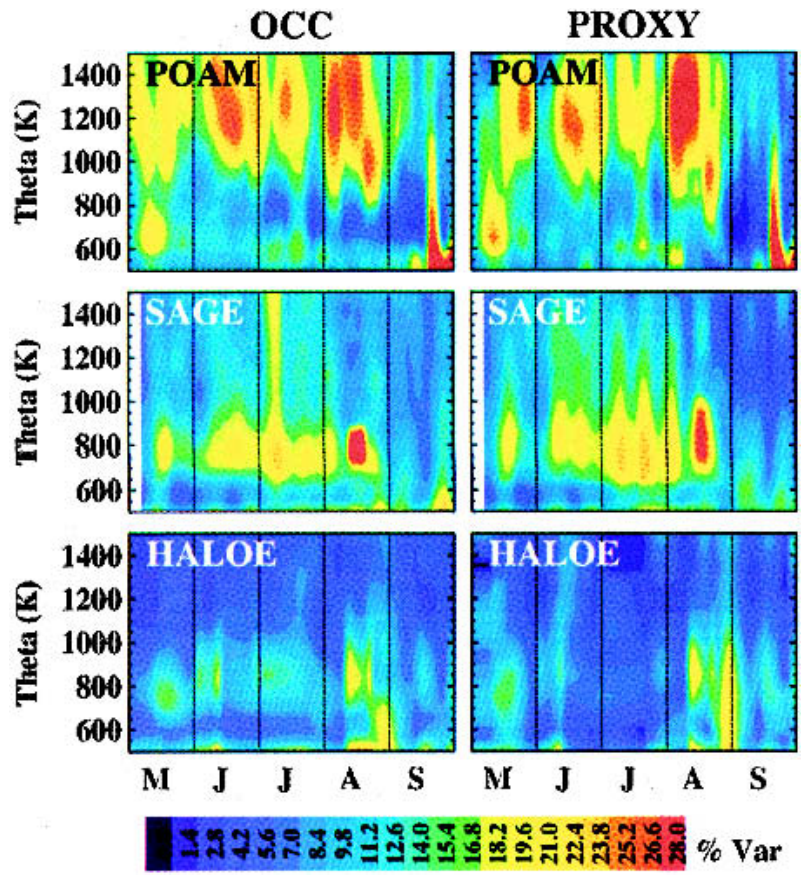

FIG. 10. Contour plot of the ozone variation at the POAM, SAGE, and HALOE measurement locations: variations in (left) the occultation data and (right) the PV proxy interpolated to the measurement locations. Ozone variation here is defined as the 7-day running standard deviation (\% relative to the ozone mixing ratio itself) of the occultation data or proxy ozone at each potential temperature. Variations from the proxy calculation have been scaled up by a factor of 1.2 for easier visualization.

\section{c. Global ozone reconstructions}

With confidence that the reconstructed proxy ozone fields are reasonable, we now use them to investigate effects of the unusual dynamical situation in 2002 on global ozone distributions. Figure 11 shows the zonal mean distribution of ozone mixing ratios derived from PV proxy calculations for the May-October time periods in 2002 and, for comparison, in 1999, at 500 ( 20 $\mathrm{km}), 850(\sim 30 \mathrm{~km})$, and $1250 \mathrm{~K}(\sim 38 \mathrm{~km})$. Steep ozone gradients marking the edge of the polar vortex are well defined at $1250 \mathrm{~K}$ in both years until mid-August. In 1999, the low-ozone vortex air stays fairly well contained at latitudes poleward of about $70^{\circ} \mathrm{S}$ into October. In 2002, however, the vortex edge gradually moves toward the pole so that by early September there is massive intrusion of low-latitude, ozone-rich air reaching the pole. At $850 \mathrm{~K}$ in 2002, subtropical air appears diluted by polar air in June-August, and after the major warming in late September low-latitude air rapidly fills the polar region. Differences between 1999 and 2002 are not as dramatic at $500 \mathrm{~K}$, consistent with previous findings that the vortex at this level remained intact and air within it remained confined during the major warming (e.g., Manney et al. 2005). Nevertheless, in September and October of 2002 ozone mixing ratios are higher than in 1999.

We next focus on the enhanced zonal ozone variability between May and September of 2002. Like Fig. 10, Fig. 12 shows running 7-day standard deviations of the proxy fields. In this case, however, hemispheric variations are shown for individual $\theta$ levels, and results are presented for both 2002 and 1999. For reference, the latitudes of the occultation measurements are overlaid on the contour plots. Black circles indicate the dates of the most significant wave events in 2002 , based on the $100-\mathrm{hPa}$ eddy heat flux, tabulated by Newman and Nash (2005). As expected, there is a strong correlation

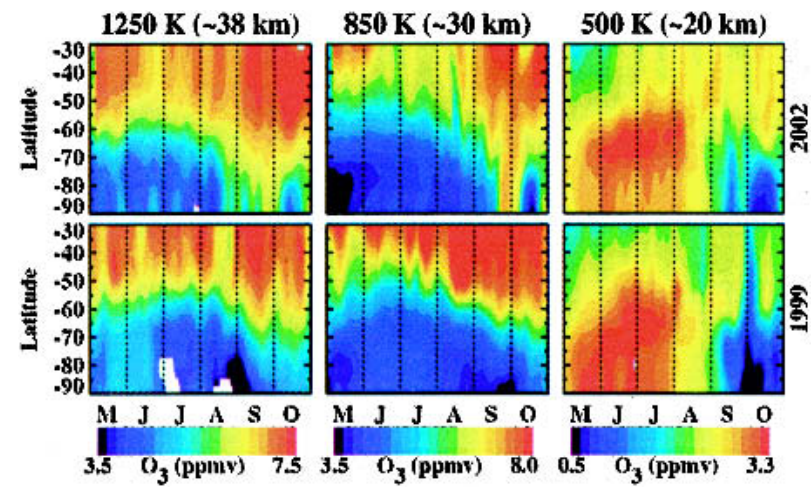

FIG. 11. Latitudinal distribution of ozone mixing ratios at potential temperatures of (left) 1250 , (center) 850 , and (right) $500 \mathrm{~K}$ in (top) 2002 and (bottom) 1999 as determined from the PV proxy. White areas denote latitude regions where the PV was outside the range of PV values encountered by the solar occultation instruments. 


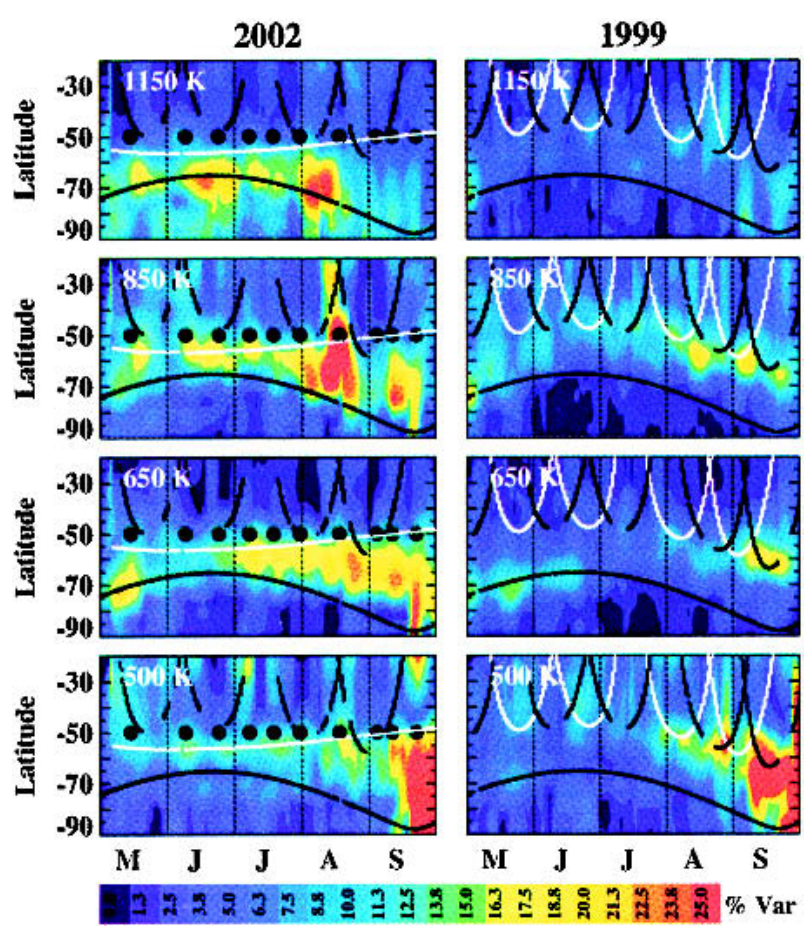

FIG. 12. Contour plots of the 7-day running standard deviation of PV proxy ozone fields from 1 May through 30 Sep in (left) 2002 and (right) 1999, for the potential temperatures noted in each panel. Solid black lines denote the latitudes of the POAM (polar) and HALOE measurements. The white line denotes the SAGE III (in 2002) and SAGE II (in 1999) measurement latitudes. Black dots signify the times of significant wave events as tabulated by Newman and Nash (2005). Variations are reported in percent relative to the average proxy ozone mixing ratios in the 7-day period.

between the timing of the wave events and increased ozone variability. In general, maximum variability in 2002 occurs between latitudes of about $50^{\circ}$ and $75^{\circ} \mathrm{S}$. In a typical year with a pole-centered vortex, this coincides with the vortex edge region, where higher variability is expected and is seen, for instance, even in 1999. The variability in 2002 is at least twice as high as in 1999, however, indicating a much stronger response of the stratosphere to wave activity in 2002, as discussed by Scaife et al. (2005).

Early winter ozone variations were larger at $1150 \mathrm{~K}$ than below, but the magnitude of the variations decreased in late August at this altitude. At $500 \mathrm{~K}$ on the other hand, perturbations coinciding with the wave events were relatively small at the beginning of the winter, escalating until reaching levels higher than $25 \%$ at the time of the major warming. The general trend is of increasing variations at successively lower altitudes as the winter progressed. This trend coincides directly with meteorological variables, such as the trend in stratospheric temperatures from $55^{\circ}$ to $75^{\circ} \mathrm{S}$, in which maximum perturbations occur above $10 \mathrm{hPa}$ in May but gradually occur at lower altitudes over the course of the winter. These and other such effects are caused by, and follow the morphology of, planetary wave energy deposition in the stratosphere, as enumerated by Newman and Nash (2005).

\section{Simulations of ozone during the major warming}

In this section, we compare the reconstructed proxy ozone fields to simulations of ozone during the major warming. We use the U.K. Universities Global Atmospheric Modeling Project (UGAMP) StratosphereMesosphere Model (USMM; Thuburn and Brugge 1994), a spectral, primitive equation model of the stratosphere and mesosphere forced at the lower boundary by prescribed geopotential heights. The USMM run used here is the "control" run of Manney et al. (2005), and the model details and configuration are described in more detail therein. This USMM configuration has a vertical resolution of about $1.6 \mathrm{~km}$, a T42 truncation (horizontal resolution of about $3^{\circ}$ ), and a lower boundary at $100 \mathrm{hPa}$, at which geopotential heights are specified from daily Met Office fields. The run was initialized using Met Office wind and temperature fields for 14 September 2002; the ozone field was initialized with the PV proxy from 14 September 2002. This proxy was constructed using a latitude-independent fit; this, in addition to the numerous interpolations involved in converting the proxy field to the model grid, results in some differences between the proxy and USMM even on the initial day. Note that the latitudeindependent proxy was used only for initialization; all other proxy calculations discussed below refer to latitude-dependent calculations unless otherwise stated.

The USMM run was unusually successful in capturing both the evolution of the flow during the major warming and the recovery from the warming (Manney et al. 2005). However, in the middle stratosphere, the recovery from the warming is slightly less complete than in observations, and the resulting modeled vortex in early October is somewhat smaller and weaker than that in the Met Office data. Manney et al. (2005) showed that enhanced poleward transport and mixing are the dominant transport processes during the warming, with enhanced diabatic descent dominating in a core region of the vortex in the lower stratosphere that remained isolated throughout the warming.

Figure 13 shows orthographic projections of the USMM simulated ozone mixing ratios at 850 (column 1) and $600 \mathrm{~K}$ (column 4) for 18, 22, 26, and 30 September and 8 October. The main features at $850 \mathrm{~K}$ of an elongated, displaced vortex breaking into two lobes that then stream out to lower latitudes is very similar to the TREL morphology discussed by Allen et al. (2003) and the PV morphology shown by Manney et al. (2005), as expected since ozone is treated as a passive tracer in the USMM. The major features at $600 \mathrm{~K}$ are similar, but the modeled vortex (low-ozone region) does not 


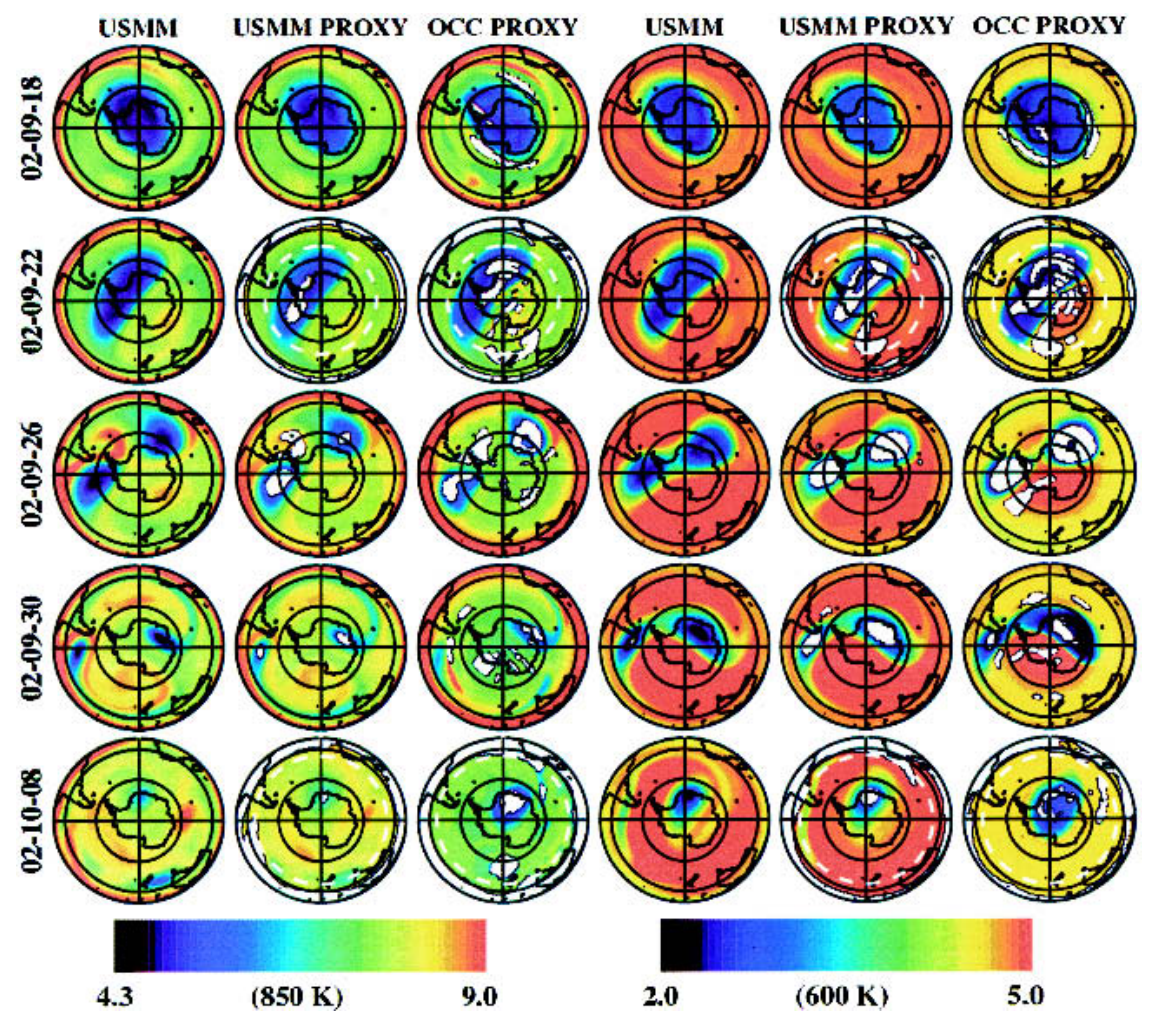

Fig. 13. Global ozone fields at $850 \mathrm{~K}$ on the dates given at the left (yy-mm-dd) from the (first column) USMM, (second column) the test proxy calculated from the USMM interpolated to the solar occultation measurement locations, and (third column) the PV proxy. (fourth, fifth, and sixth columns) Analogous maps except for $600 \mathrm{~K}$. Projections are orthographic with $0^{\circ}$ at the top and $90^{\circ} \mathrm{E}$ at the right; latitude lines are drawn at the $0^{\circ}, 30^{\circ}$, and $60^{\circ} \mathrm{S}$. White areas denote those regions where PV values were outside the range sampled by the solar occultation instruments. Dashed white lines, when present, designate the most equatorward latitude of the data included in the proxy calculation. The color bars refer to ozone mixing ratio in ppmv.

completely split, and the tongues of material pulled off the vortex are smaller and confined to ozone values characteristic of those near the vortex edge region. To aid in interpretation of differences between the proxy and USMM fields, a test proxy ozone field has been reconstructed from the USMM ozone and PV fields interpolated to the solar occultation measurement locations (the "USMM proxy"; Fig. 13, columns 2 and 5). This simulates the effects of errors in the reconstruction technique related to the limited sampling of the occultation instruments, degrading the USMM field according to these errors. The PV proxy from occultation (OCC) measurements ("OCC proxy") is shown in Fig. 13 (columns 3 and 6). Comparisons among the USMM fields, the USMM proxy, and the OCC proxy can help distinguish differences that are related to sampling from those related to misrepresentation, or lack of representation, of physical processes in the reconstruction or the USMM.

The most obvious USMM modeling error is the omission of photochemistry, especially in middle- and upper-stratosphere regions where air rapidly changes lati- tude and then remains confined at the new latitude for several days. At $850 \mathrm{~K}$, the USMM and proxy fields agree in the vortex region on 18 through 30 September, during which time ozone in the vortex increases, consistent with the mixing in of extravortex air indicated in long-lived tracer calculations (Manney et al. 2005). By 8 October, however, when the remaining vortex has for several days been located once again at high latitudes, the OCC proxy vortex ozone is considerably lower, consistent with relaxation toward photochemical equilibrium values characteristic of that latitude, a process not represented in the USMM fields (that the low-ozone vortex region is smaller in the USMM fields on 30 October is related to the imperfect representation in the model of the vortex recovery, as noted above). In addition, the lower equatorial ozone values in the USMM on 26 and 30 September are directly related to the model's lack of an ozone source in that region.

The most obvious PV-mapping errors result when the $\mathrm{PV}-\mathrm{O}_{3}$ fitting improperly accounts for photochemistry (or lack thereof) of air parcels in latitude regions 
not sampled by the occultation instruments, from errors in the PV fields and from sparse sampling by the occultation instruments. Such errors are apparent in the OCC proxy on 26 and 30 September. Here, the highlatitude proxy fields outside the vortex fail to capture the morphology that is expected from the very rapid transport of low-latitude, high ozone air into the polar regions at this time. For instance, the OCC proxy on 26 September gives little indication of the hook of highozone air between South America and Antarctica. Instead, the OCC proxy fields are more zonally symmetric, and the distinction between vortex and surrounding air is obscured. In the TREL proxy, on the other hand (not shown), the hook of high-ozone air on 26 September is apparent, and the fields are much less zonally symmetric, indicating that errors in the PV fields are contributing to the unrealistic OCC proxy morphology. There are differences between even the TREL proxy and USMM, however, that arise from the approximation that the $\mathrm{PV}-\mathrm{O}_{3}$ (or TREL- $\mathrm{O}_{3}$ ) relation varies linearly with latitude. That is, low-latitude air pulled up to higher latitudes is assigned some of the characteristics of the already equilibrated high-latitude air in the latitude-dependent fitting procedure. This results in an underestimate of ozone in those tongues of air, even when they in fact have not had time to equilibrate to the high-latitude conditions.

Comparison of the USMM, USMM proxy, and OCC proxy fields shows some examples of features that are not captured in the OCC proxy because of the sparse sampling. For example, on 26 September at $850 \mathrm{~K}$, the USMM proxy truncates the tongues of very low ozone pulled off each vortex, probably indicating inadequate sampling of those regions. Other cases are not as clear cut; for instance, the long tongues of vortex material pulled out on 30 September are captured well in the USMM proxy but are not as apparent in the OCC proxy. Since these tongues are at quite low latitude, lack of chemistry in the USMM might also contribute to the discrepancies. Comparison of $\mathrm{H}_{2} \mathrm{O}$ transported in the USMM with OCC proxy $\mathrm{H}_{2} \mathrm{O}$ (not shown) shows quite good agreement between the tongue of high $\mathrm{H}_{2} \mathrm{O}$ pulled off the vortex lobe near $270^{\circ} \mathrm{E}$ in the USMM and in proxy fields based on both PV and TREL reconstructions, as well as evidence in the proxy fields for a tongue pulled off the other vortex that does not extend quite as far as that in the USMM. This indicates that the dynamical features are correctly captured in both the USMM and OCC proxy, and the question of disagreements in the USMM and OCC proxy fields is a matter of the extent to which photochemistry altered ozone in these dynamical features and the extent to which this chemistry is correctly captured in the reconstruction.

At $600 \mathrm{~K}$, dynamical time scales are expected to be substantially faster than chemical time scales, and thus neither the lack of chemistry in the USMM nor the limitations of the latitude-dependent fitting are ex- pected to be significant factors. Agreement between the USMM and OCC proxy fields at $600 \mathrm{~K}$ is excellent during 18-30 September, apart from a small high bias in the USMM and USMM proxy in the extravortex region carried over from a similar difference in the initial fields on 14 September. On 8 October, the USMM calculation defines a significantly smaller low-ozone region than the OCC proxy, caused by the underestimated size and strength of the recovery of the modeled vortex (Manney et al. 2005). However, the ozone values in the vortex agree quite well between the USMM and proxy. On 30 September and 8 October, the USMM proxy reproduces fairly well the narrow tongues of low ozone pulled off from the edge region of both lobes of the vortex. These tongues are not as distinct, however, in either the OCC PV or higher resolution TREL proxy fields, which may indicate that the air in them has begun to photochemically equilibrate to the lowerlatitude conditions; detailed analysis of chemistry along the trajectories of these air parcels is necessary to test this hypothesis. The increase in ozone outside the vortex is related primarily to enhanced diabatic descent in the anticyclone region at this level, as noted in USMM results by Manney et al. (2005) and confirmed by highresolution 3D Lagrangian transport calculations using Met Office winds and temperatures and a middleatmosphere radiation code (not shown). The overall decrease seen in the OCC proxy at midlatitudes between 30 September and 8 October is at present unexplained, and further investigation of the sampling and reconstruction methods, as well as the robustness of the transport calculations is needed to address this discrepancy.

Figure 14 gives an overview of the large-scale changes in USMM and OCC proxy ozone during the period of the major warming. As shown by Manney et al. (2005) for long-lived tracers, the dominant transport effect in the mid- to high EL stratosphere is enhanced poleward transport and mixing of extravortex air into

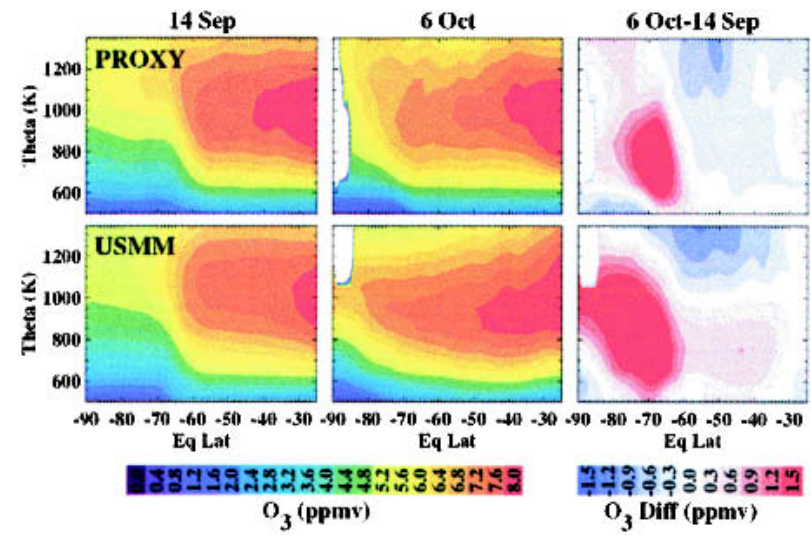

FIG. 14. EL/ $\theta$ contour plots of the (top) PV proxy and (bottom) USMM on (left) 14 Sep, (center) 6 Oct, and (right) the differences between them. 
the vortex region. The exception is in the lowerstratospheric vortex core, which remains well isolated; enhanced descent is the dominant transport process here. Figure 14 (bottom) shows how these transport processes alone are expected to affect ozone, with large increases in nearly all of the middle and high EL stratosphere. Comparison with the OCC proxy shows very similar increases below about $1100 \mathrm{~K}$ and poleward of about $60^{\circ} \mathrm{S}$ EL. This good agreement (which is robust for any selection of ending date near this time) indicates that these transport processes, rather than photochemical effects not represented in the USMM, are the primary factor driving ozone changes through much of the stratosphere during the major warming. Disagreements between the proxy and USMM above $1100 \mathrm{~K}$ in the polar region are most likely caused by the lack of photochemistry in the USMM, in which air transported from lower latitudes is not allowed to relax to the equilibrium (lower ozone) conditions of the polar region on 6 October.

\section{Discussion}

The unique dynamical situation in 2002 captured the attention of the public because of its profound effects on the ozone hole, as defined by column measurements made by the Total Ozone Mapping Spectrometer (TOMS) and Global Ozone Monitoring Experiment (GOME) satellite instruments (Weber et al. 2003; Sinnhuber et al. 2003). As early as the first press releases announcing the precipitous drop in ozone hole area, it was well understood that the near disappearance of the ozone hole was due almost entirely to dynamical processes. As explained by Hoppel et al. (2003) and Allen et al. (2003), the primary reason that the ozone hole was so diminished in size was that the area of chemical ozone depletion in the lower stratosphere was overlaid by areas of high-ozone intrusions from lower latitudes in the middle and upper stratosphere. In this section, we use the proxy ozone fields to illustrate the vertical distribution of global ozone, showing the morphology that led to such dramatic increases in column ozone.

Figure 15 depicts the PV proxy ozone fields from 500 (about $20 \mathrm{~km}$ ) to $1350 \mathrm{~K}$ (about $40 \mathrm{~km}$ ) on 22, 26, and 30 September and 8 October in 2002. In a typical year, the region of low ozone at all of these altitudes is contained within the vortex, which sits symmetrically over the pole throughout this time period. At altitudes below $550 \mathrm{~K}$, ozone inside the vortex is reduced by photochemical depletion; without depletion (as is often the case in the $\mathrm{NH}$ ), ozone would be higher inside the vortex than outside at the lowest altitude depicted here. At the higher altitudes, ozone inside the vortex is low for the reasons stated above regarding latitude gradients and vortex containment. In 2002 there were still large regions of low, chemically depleted ozone inside the vortex at $500 \mathrm{~K}$ (and below), although not as large or as symmetric as in a typical year. The more significant differences from a typical year, however, are seen in the ozone distributions from 650 to $1350 \mathrm{~K}$. On 22 September, the regions of low ozone at these altitudes are shifted away from the pole, displaced by a large area of ozone-rich air that has been transported from lower latitudes. This situation intensifies throughout September as the vortex pulls apart in the middle and upper stratosphere, causing the vortex air to mix with surrounding air and leaving vast areas of ozone-rich air in the polar region.

This situation is compared to 1999 , a typical year, in Fig. 16. Here longitude/ $\theta$ cross sections of ozone mixing ratio profiles at $75^{\circ} \mathrm{S}$ from the USMM simulation and PV proxy in 2002 are compared to the PV proxy from 1999 on 18 and 26 September. The 1999 proxy included SAGE II data, but not SAGE III data. Qualitatively, the USMM and proxy fields in 2002 are similar, but are very different from the PV proxy in 1999. In 1999, the fields are symmetric, with EL higher than $55^{\circ} \mathrm{S}$ at all altitudes on both dates. In 2002, there is much less symmetry, and significantly more air of low EL. By 26 September, the 2002 ozone fields show a pronounced westward tilt with increasing altitude up to about $800 \mathrm{~K}$ for the vortex lobe near $300^{\circ} \mathrm{E}$ longitude. Above this altitude, the proxy fields show little evidence of a distinct vortex, instead simply showing the pervasive signature of low-latitude, ozone-rich air. The USMM shows a region of relatively low ozone corresponding to high-EL air at the higher altitudes. Examination of 3D model fields indicates that this arises mainly from a vortex fragment in the USMM simulation that is slightly displaced, and stronger, than that seen in the Met Office PV (note that the proxy does show a region of slightly lower ozone here); the absence of chemical processes in the USMM may also contribute to the appearance of this low-ozone region.

A nadir-looking instrument such as TOMS or GOME would observe directly through these regions of high-ozone air above $800 \mathrm{~K}$ and would intersect the tilted region of low-ozone air below, effectively masking any photochemical depletion occurring in the lower stratosphere. This is illustrated in Fig. 17, where we show partial columns from the PV proxy on 22,26 , and 30 September and 8 October. The partial column above the conventional ozone hole region is shown in Fig. 17 (top), corresponding to theta surfaces from 600 to 1900 $\mathrm{K}$ (the top of the proxy calculation) or altitudes from about 22 to $45 \mathrm{~km}$. This should be compared to the second row, which shows the partial column over the primary region of photochemical ozone depletion, from 350 to $600 \mathrm{~K}$ or about 13 to $22 \mathrm{~km}$. The bottom two rows show the total proxy column from 350 to $1900 \mathrm{~K}$ and the TOMS total column observations. Because the proxy calculation lower boundary is at $350 \mathrm{~K}$, it underestimates the actual total column by an amount approximately equal to the tropospheric (and lowermost stratosphere) column. To account for this, the color 

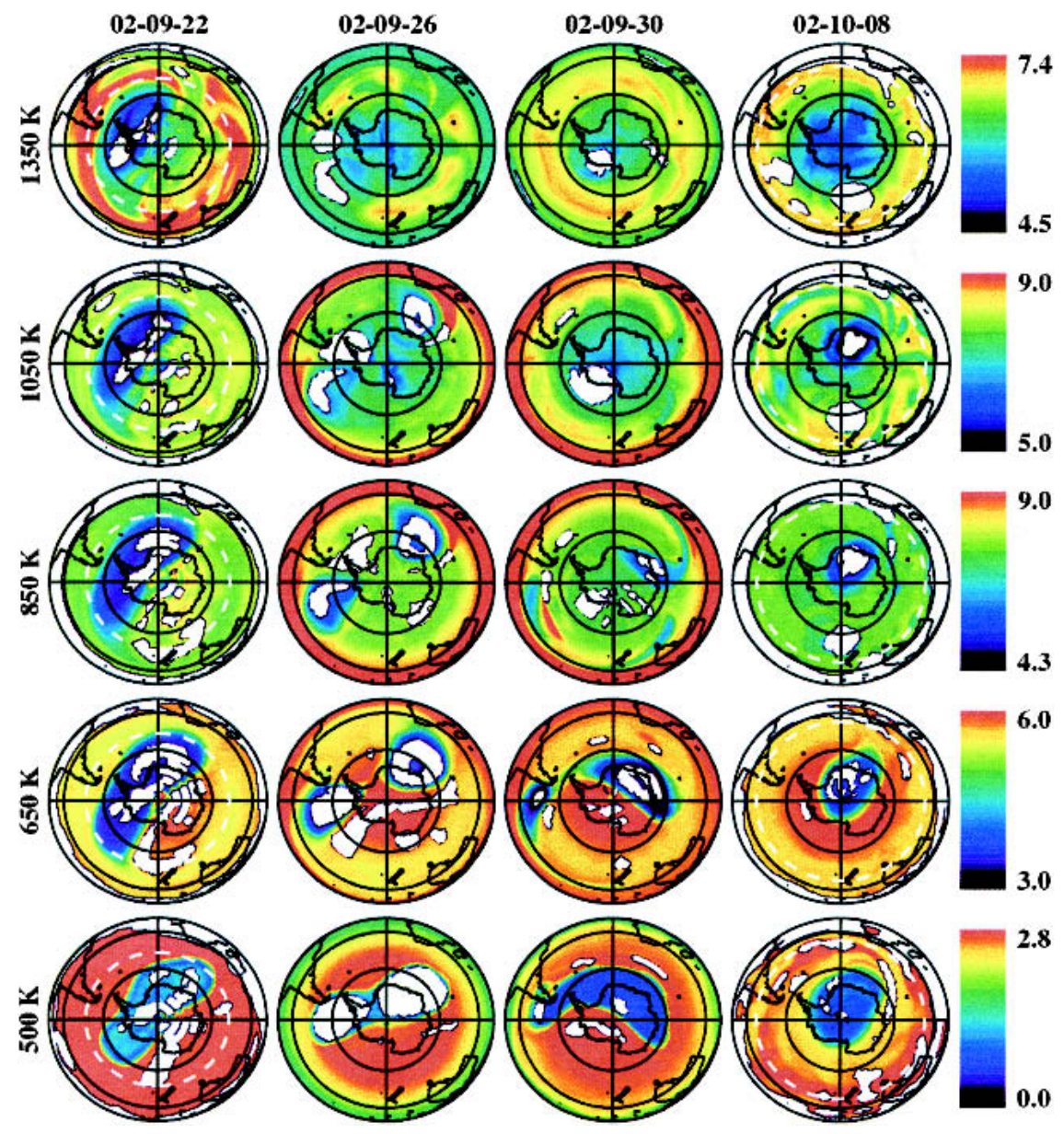

FIG. 15. Orthographic projections of the PV proxy ozone fields (as in Fig. 13) at the theta levels listed on the left and for the dates (yy-mm-dd) listed at the top of each column. The color bars on the right refer to ozone mixing ratio (ppmv).

scale for the 350-1900-K proxy maps has been shifted by 50 Dobson units (DU) relative to the color scale applied to the TOMS maps. The PV proxy column agrees exceptionally well with observed TOMS columns. The region of photochemically depleted air, as indicated by the $350-600-\mathrm{K}$ column, is extensive on all of the dates depicted here. Disruption of the vortex at higher altitudes, however, leads to unusually high column ozone from 600 to $1900 \mathrm{~K}$ in the polar region. The total proxy column from 350 to $1900 \mathrm{~K}$ of course reflects a combination of the morphology of the two partial columns described here, resulting in a much smaller region of low-column ozone values than indicated by the region of photochemical depletion.

\section{Summary and conclusions}

We have described the effects of unusual dynamical activity in the $2002 \mathrm{SH}$ winter on stratospheric ozone measurements from the POAM III, SAGE III, and HALOE solar occultation instruments. These effects

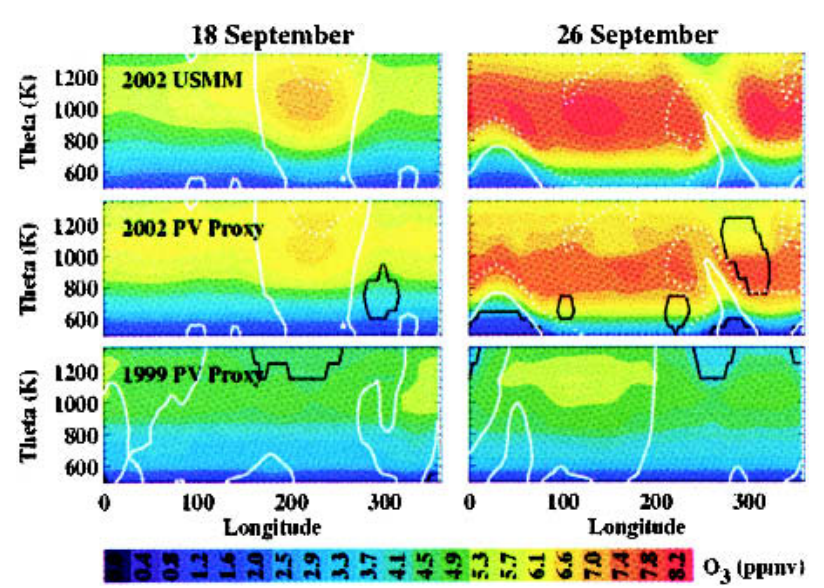

FIG. 16. Cross sections at $75^{\circ} \mathrm{S}$ latitude of ozone mixing ratio profiles from the (top) USMM simulation and (middle) PV proxy in 2002, and (bottom) from the PV proxy in 1999 for (left) 18 and (right) 26 Sep. Black solid lines denote areas inside of which the PV was not sampled by the solar occultation instruments. White contours denote EL of -75 (solid) and -55 (dotted). 

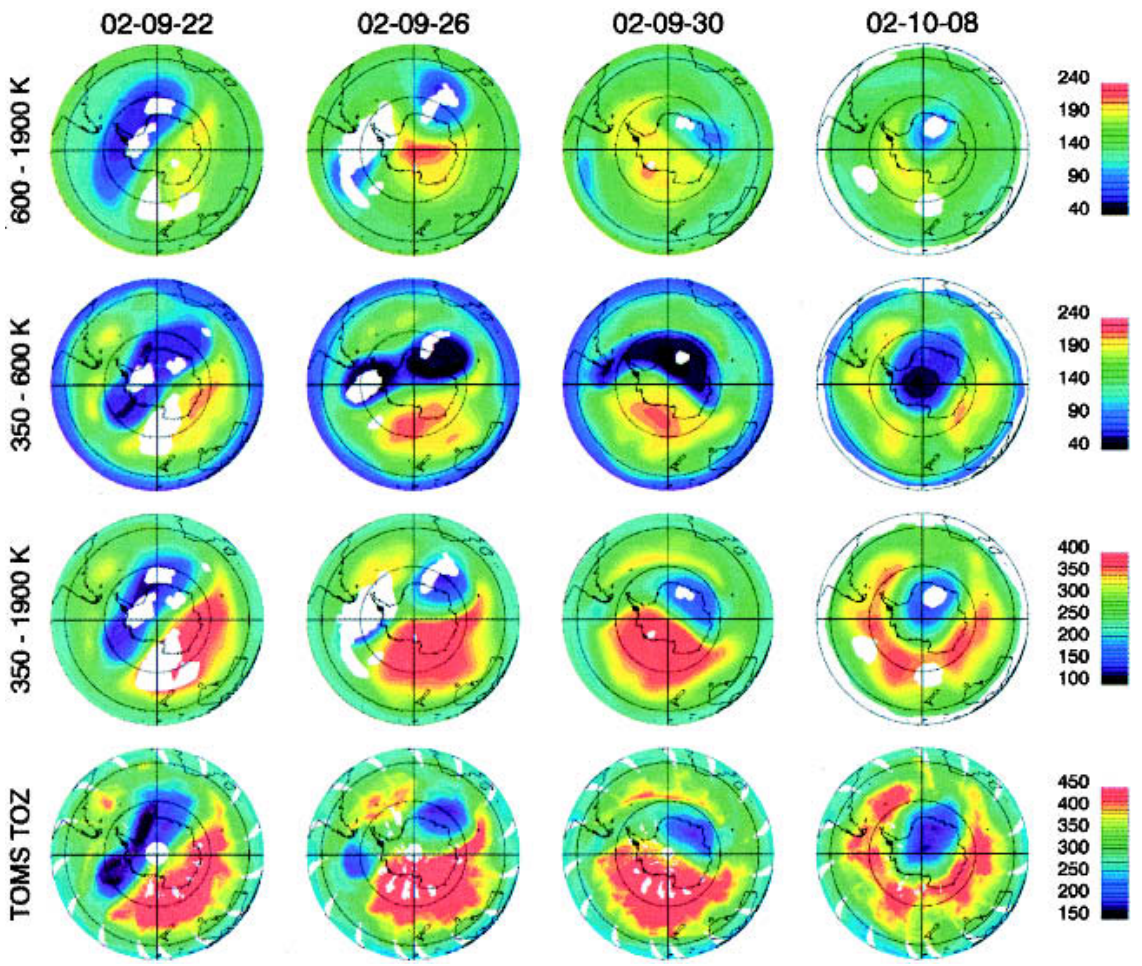

FIG. 17. Partial column ozone from the PV proxy over the altitude range from (top row) 600 to $1900 \mathrm{~K}$ (above about $22 \mathrm{~km}$ ), (row 2) 350 to $600 \mathrm{~K}$ (from about 13-22 km), and (row 3$) 350$ to $1900 \mathrm{~K}$ on the dates listed at the top. (row 4) TOMS column ozone. The color scale for row 3 has been shifted by 50 DU relative to that for the TOMS data to account for the missing ozone from the surface to $350 \mathrm{~K}$ in the proxy calculations. White areas denote regions of missing data.

are largely manifested by increased variability in the ozone data throughout the winter caused by the sampling of air with larger-than-normal ranges of EL. In late September, POAM measurements are located at latitudes poleward of $85^{\circ} \mathrm{S}$ and, until 2002, always occurred in the region of maximum ozone depletion. Rapid transport of low-latitude air to the polar region during the late-September 2002 major warming resulted in POAM observations of ozone-rich air in this normally chemically depleted region. At the same time, the SAGE III measurements located near $48^{\circ} \mathrm{S}$ showed clear indications of ozone-depleted air in the lower stratosphere.

Three-dimensional, global ozone distributions were reconstructed from the solar occultation data using a PV-mapping technique. These proxy ozone fields were evaluated by comparisons of the solar occultation data on which they were based with ozonesondes at Lauder, New Zealand, and the South Pole, with a mechanistic model simulation and with total column data from the TOMS instrument. Global ozone distributions in 2002 were compared to those in 1999, revealing substantial differences in the zonal average ozone mixing ratios, particularly in the August-September time period, and dramatically increased variability in 2002 near the vor- tex edge region. Unusually large, episodic increases in ozone variability as early as May correlate directly with the occurrence of wave events forced from the troposphere. This supports the idea that dynamical forcing early in the 2002 winter influenced the morphology of the stratosphere in a significant and unusual manner, possibly setting the stage for the unprecedented major stratospheric warming in late September. The global reconstructions compare well with a mechanistic model that treats ozone as a passive tracer in regions where photochemistry is negligible. At altitudes where photochemistry is relevant, differences between the model and proxy ozone fields are consistent with the effects expected from chemical production and loss processes and from limitations in the PV-mapping procedure.

Effects of the increased dynamical activity in 2002 on column ozone measurements were quantified by examining the global distribution of partial column ozone over altitudes both above and within the main region of photochemical ozone depletion. After the major warming in late September, areas of significant ozone depletion in the lower stratosphere were overlaid by areas of ozone-rich air transported from lower latitudes to the polar region, resulting in total column values that were much higher than normally found in the presence of 
chemically depleted air. When normalized for the lack of tropospheric data in the proxy calculations, global total column distributions based on the proxy show excellent agreement with column observations from the TOMS satellite. The proxy calculations thus constitute an effective means for understanding the processes underlying the column measurements.

Nearly two decades of solar occultation ozone measurements are currently available, providing a rich database of very precise, trend-quality, high-verticalresolution ozone profiles. Ultimately, these data should be formally assimilated into a comprehensive, longterm observational database. The work presented here represents the most extensive effort yet at combining these datasets in a scientifically legitimate manner. The reconstructed ozone fields provide an exceptional view of the global ozone distributions throughout the 2002 $\mathrm{SH}$ winter and during the unprecedented major warming in late September. In principle, the PV-mapping approach can be further modified to address some of its shortcomings, for instance, by including components such as photochemistry along air parcel trajectories to address solar insolation dependence more rigorously. Fundamentally, PV-mapping is less capable than formal data assimilation, but the results presented here suggest that it can be highly effective for scientific investigations of stratospheric ozone processes.

Acknowledgments. C.E.R. was supported by the Naval Research Laboratory Award N00173-01-01-G007. We thank A. O'Neill for contributions to the USMM modeling effort. Work at the Jet Propulsion Laboratory, California Institute of Technology was done under contract with the National Aeronautics and Space Administration. W.A.L. is at the Data Assimilation Research Centre (DARC), funded by the UK National Environment Research Council (NERC). Ozonesonde balloons were launched at the South Pole by Loreen Lock and Brian Vase of the NOAA Climate Monitoring and Diagnostics Laboratory.

\section{REFERENCES}

Allaart, M. A. F., H. Kelder, and L. C. Heijboer, 1993: On the relation between ozone and potential vorticity. Geophys. Res. Lett., 20, 811-814.

Allen, D. R., and N. Nakamura, 2001: A seasonal climatology of effective diffusivity in the stratosphere. J. Geophys. Res., 106, 7917-7935.

- , and - 2002: Dynamical reconstruction of the record low column ozone over Europe on 30 November 1999. Geophys. Res. Lett., 29, 1362, doi:10.1029/2002GL014935.

$\longrightarrow$, and — 2003: Tracer equivalent latitude: A diagnostic tool for isentropic transport studies. J. Atmos. Sci., 60, 287-304.

-, R. M. Bevilacqua, G. E. Nedoluha, C. E. Randall, and G. L. Manney, 2003: Unusual stratospheric transport and mixing during the 2002 Antarctic winter. Geophys. Res. Lett., 30, 1599, doi:10.1029/2003GL017117.

Brühl, C., and Coauthors, 1996: Halogen Occultation Experiment ozone channel validation. J. Geophys. Res., 101, $10217-$ 10240 .
Butchart, N., and E. E. Remsberg, 1986: The area of the stratospheric polar vortex as a diagnostic for tracer transport on an isentropic surface. J. Atmos. Sci., 43, 1319-1339.

Charlton, A. J., A. O'Neill, W. A. Lahoz, P. Berrisford, P. van Velthoven, H. Eskes, and H. Kelder, 2005: The splitting of the stratospheric polar vortex in the Southern Hemisphere, September 2002: Dynamical evolution. J. Atmos. Sci., 62, 590-602.

Cunnold, D. M., W. P. Chu, R. A. Barnes, M. P. McCormick, and R. E. Veiga, 1989: Validation of SAGE II ozone measurements. J. Geophys. Res., 94, 8447-8460.

Haynes, P. H., and E. Shuckburgh, 2000: Effective diffusivity as a diagnostic of atmospheric transport, 1. Stratosphere. J. Geophys. Res., 105, 22 777-22 794.

Hoppel, K. W., R. M. Bevilacqua, D. R. Allen, G. E. Nedoluha, and C. E. Randall, 2003: POAM III observations of the anomalous 2002 Antarctic ozone hole. Geophys. Res. Lett., 30, 1394, doi:10.1029/2003GL016899.

Lary, D. J., M. P. Chipperfield, J. A. Pyle, W. A. Norton, and L. P. Riishojgaard, 1995: Three-dimensional tracer initialization and general diagnostics using equivalent PV latitudepotential-temperature coordinates. Quart. J. Roy. Meteor. Soc., 121, 187-210.

Leovy, C. B., C.-R. Sun, M. H. Hitchman, E. E. Remsberg, J. M. Russell III, L. L. Gordley, J. C. Gille, and L. V. Lyjak, 1985: Transport of ozone in the middle stratosphere: Evidence for planetary wave breaking. J. Atmos. Sci., 42, 230-244.

Manney, G. L., J. D. Farrara, and C. R. Mechoso, 1994: Simulations of the February 1979 stratospheric sudden warming: Model comparisons and three-dimensional evolution. Mon. Wea. Rev., 122, 1115-1140.

—, L. Froidevaux, J. W. Waters, and R. W. Zurek, 1995: Evolution of Microwave Limb Sounder ozone and the polar vortex during winter. J. Geophys. Res., 100, 2953-2972.

, H. A. Mickelsen, M. L. Santee, M. R. Gunson, F. W. Irion, A. E. Roche, and N. J. Livesey, 1999: Polar vortex dynamics during spring and fall diagnosed using trace gas observations from the Atmospheric Trace Molecule Spectroscopy instrument. J. Geophys. Res., 104, 18 841-18 866.

- and Coauthors, 2005: Simulations of dynamics and transport during the September 2002 Antarctic major warming. J. Atmos. Sci., 62, 690-707.

Morris, G. A., J. F. Gleason, J. M. Russell III, M. R. Schoeberl, and M. P. McCormick, 2002: A comparison of HALOE v19 with SAGE II v6.00 ozone observations using trajectory mapping. J. Geophys. Res., 107, 4177, doi:10.1029/2001JD000847.

Nedoluha, G. E., R. M. Bevilacqua, M. D. Fromm, K. W. Hoppel, and D. R. Allen, 2003: POAM measurements of PSCs and water vapor in the 2002 Antarctic vortex. Geophys. Res. Lett., 30, 1796, doi:10.1029/2003GL017577.

Newman, P. A., and E. R. Nash, 2005: The unusual Southern Hemisphere stratosphere winter of 2002. J. Atmos. Sci., 62, 614-628.

Orsolini, Y. J., C. E. Randall, G. L. Manney, and D. R. Allen, 2005: An observational study of the final breakdown of the Southern Hemisphere stratospheric vortex in 2002. J. Atmos. Sci., 62, 735-747.

Randall, C. E., and Coauthors, 1995: Preliminary results from POAM II: Stratospheric ozone at high northern latitudes. Geophys. Res. Lett., 22, 2733-2736.

— , and Coauthors, 2002: Reconstruction of three-dimensional ozone fields using POAM III during SOLVE. J. Geophys. Res., 107, 8299, doi:10.1029/2001JD000471.

— parisons with ozonesonde and satellite data. J. Geophys. Res., 108, 4367, doi:10.1029/2002JD002944.

Scaife, A. A., D. R. Jackson, R. Swinbank, N. Butchart, H. E. 
Thornton, M. Keil, and L. Henderson, 2005: Stratospheric vacillations and the major warming over Antarctica in 2002. J. Atmos. Sci., 62, 629-639.

Schoeberl, M. R., and Coauthors, 1989: Reconstruction of the constituent distribution and trends in the Antarctic polar vortex from ER-2 flight observations. J. Geophys. Res., 94, $16815-16845$.

Sinnhuber, B.-M., M. Weber, A. Amankwah, and J. P. Burrows, 2003: Total ozone during the unusual Antarctic winter of 2002. Geophys. Res. Lett., 30, 1580 doi:10.1029/ 2002GL016798.

Struthers, H., R. Brugge, W. A. Lahoz, A. O’Neill, and R. Swin- bank, 2002: Assimilation of ozone profiles and total column measurements into a global general circulation model. $J$. Geophys. Res., 107, 4438, doi:10.1029/2001JD000957.

Swinbank, R., and A. O’Neill, 1994: A stratosphere-troposphere data assimilation system. Mon. Wea. Rev., 122, 686-702.

Thuburn, J., and R. Brugge, 1994: The UGAMP stratosphere and mesophere model. UGAMP Internal Rep. 34.

Weber, M., S. Dhomse, F. Wittrock, A. Richter, B.-M. Sinnhuber, and J. P. Burrows, 2003: Dynamical control of $\mathrm{NH}$ and $\mathrm{SH}$ winter/spring total ozone from GOME observations in 19952002. Geophys. Res. Lett., 30, 1583, doi:10.1029/ 2002GL016799. 